\section{$\underset{\substack{\text { hommes } \\ \text { \& migrations }}}{ }$}

\section{Hommes \& migrations}

Revue française de référence sur les dynamiques

migratoires

$1319 \mid 2017$

Réfugiés et migrants au Liban

\title{
Santé, éducation, naissances
}

Quelques données de base sur la situation des réfugiés syriens au Liban

\section{Houda Kassatly}

\section{Q OpenEdition \\ 1 Journals}

\section{Édition électronique}

URL : http://journals.openedition.org/hommesmigrations/3985

DOI : ERREUR PDO dans /localdata/www-bin/Core/Core/Db/Db.class.php L.34 : SQLSTATE[HY000]

[2006] MySQL server has gone away

ISSN : 2262-3353

Éditeur

Musée national de l'histoire de l'immigration

Édition imprimée

Date de publication : 1 octobre 2017

Pagination : 119-123

ISBN : 978-2-919040-39-1

ISSN : $1142-852 X$

\section{Référence électronique}

Houda Kassatly, «Santé, éducation, naissances », Hommes \& migrations [En ligne], 1319| 2017, mis en ligne le 01 octobre 2020, consulté le 07 janvier 2021. URL : http://journals.openedition.org/ hommesmigrations/3985; DOI : https://doi.org/ERREUR PDO dans /localdata/www-bin/Core/Core/ Db/Db.class.php L.34 : SQLSTATE[HY000] [2006] MySQL server has gone away 


\title{
SANTÉ, ÉDUCATION, NAISSANCES \\ QUELQUES DONNÉES DE BASE SUR LA SITUATION DES RÉFUGIÉS SYRIENS AU LIBAN
}

\author{
Par HOUDA KASSATLY, anthropologue photographe, chercheure associée à l'Unité \\ interdisciplinaire de recherche sur la mémoire, CEMAM, université Saint-Joseph (Beyrouth) et \\ FILIPPO MARIA MARRANCONI, anthropologue, doctorant à l'EHESS (LAU).
}

\section{Couverture de santé}

Les réfugiés syriens enregistrés auprès du Haut Commissariat des Nations unies pour les réfugiés (HCR) bénéficient d'une couverture de santé partielle. Ils accèdent aux services de santé primaire par le réseau des centres du ministère de la Santé publique et du ministère des Affaires sociales, par les centres et les dispensaires gérés par les organisations non gouvernementales (ONG), souvent partenaires du HCR et du ministère de la Santé ${ }^{1}$. La population déplacée syrienne se tourne beaucoup plus que la population libanaise vers les centres de santé primaire : environ $50 \%$ des visites médicales sont, en effet, exécutées dans le cadre des services de santé primaire, tandis que la population libanaise privilégie le secteur privé non conventionné ${ }^{2}$.

Le coût d'une consultation médicale dans ces centres varie entre 3000 et 5000 livres libanaises. Cependant, dans un grand nombre d'entre eux, d'autres services sont donnés à titre gratuit aux populations considérées comme les plus vulnérables. C'est le cas des vaccinations, des services de santé prénatale et natale, et des médicaments compris dans le programme d'aide du Young Men's Christian Association (YMCA). Les tests diagnostiques et de laboratoire, les radiographies et d'autres examens nécessaires à la poursuite du traitement ou à l'hospitalisation sont aux frais de la personne. Le HCR couvre $85 \%$ des dépenses des personnes handicapées, des femmes enceintes, des enfants de moins de 5 ans et des adultes de plus de 60 ans $^{3}$.

L'accès à la santé secondaire et tertiaire, majoritairement privé, peut être effectué par tout individu, mais à ses propres frais. Pour les réfugiés, le HCR a établi des conventions avec 53 hôpitaux, publics et privés. Celles-ci stipulent que les frais d'hospitalisation sont couverts à 75 \% (les $25 \%$ restants sont à la charge des usagers), mais seulement dans le cas d'un danger pour la vie de la personne, en cas accouchement ou de soins pour le nouveau-né; et à condition que le pronostic soit positif ${ }^{4}$. La personne enregistrée au HCR doit, afin d'accéder à ce type de service, passer prioritairement par les centres de santé primaire et être orientée vers l'hôpital par ces derniers. Elle doit être en possession d'un document d'identité valide ou du certificat d'enregistrement au HCR.

Pour les situations jugées particulièrement problématiques: familles extrêmement vulnérables, enfants ayant besoin de soins intensifs, cas d'hospitalisation psychiatrique, la couverture médicale peut atteindre les $90 \%$. Pour les victimes de viol, de torture ou de violence de genre, la couverture atteint les $100 \%$.

Les maladies chroniques comme le diabète, l'épilepsie ou les problèmes cardiaques sont pris en charge 
dans les centres de santé primaire qui fournissent les médicaments adéquats. Il n'en va pas de même pour les maladies chroniques qui demandent une hospitalisation réitérée, comme les cancers, la thalassémie ou la dialyse rénale, pour lesquels il n'y a aucun type de couverture.

Le $\mathrm{HCR}$ privilégie une approche en santé publique qui recoupe le système libanais où l'accès aux services de santé est déterminé par le statut social, économique, juridique et politique des patients. Cependant, les politiques du secteur humanitaire n'arrivent pas à répondre aux besoins primaires de la population réfugiée, et le système de protection sociale ne paraît pas capable de
Les frais demeurent donc

l'un des obstacles majeurs de

l'accès aux services : dans les six derniers mois de 2016, le HCR estime que la moitié des familles s'est adressée aux centres de santé primaire, mais que $16 \%$ d'entre elles n'ont pas pu bénéficier de leurs services. réduire sa pauvreté 6 . Les frais demeurent donc l'un des obstacles majeurs de l'accès aux services : dans les six derniers mois de 2016, le HCR estime que la moitié des familles s'est adressée aux centres de santé primaire, mais que $16 \%$ d'entre elles n'ont pas pu bénéficier de leurs services. Parmi celles-ci, $94 \%$ en ont été empêchées par le coût du traitement, $14 \%$ par le coût du transport, et $17 \%$ ont été refusées par l'institution. Ces estimations varient selon les régions du Liban?.

Si le coût est un obstacle majeur à l'accès aux services de santé pour la population déplacée, dont la situation financière est déjà très défavorable, d'autres facteurs difficilement quantifiables contribuent à en entraver l'accès. Comme le montrent Parkinson et Behrouan pour le Liban, les politiques de santé, l'instabilité financière, les conditions de séjour, la présence de barrages et de contrôles de sécurité, la fragmentation géographique des familles, ainsi que les arrangements concrets que les personnes doivent quotidiennement effectuer, créent des hiérarchies d'éligibilité aux services qui doivent être étudiées de manière située ${ }^{8}$.

Selon un rapport d'Amnesty International, citant le $\mathrm{HCR}$, sur les $24 \%$ de réfugiés inscrits à l'agence onusienne qui ont regagné la Syrie, 11 \% d'entre eux a été dicté par des raisons médicales 9 .

\section{Éducation}

Six ans après le du début de la crise syrienne, à peu près 250000 enfants réfugiés en âge de scolarisation (3-18 ans) - sur les 495910 enfants syriens réfugiés enregistrés au HCR - ont accès au système scolaire $^{10}$. L'accès à l'éducation est donc assuré à $50 \%$ environ des enfants syriens quand, en Syrie, le pourcentage d'accès à l'école primaire s'élevait à $90 \%{ }^{11}$.

Le système scolaire libanais est composite : face à un secteur privé prépondérant (payant ou gratuit), le système public reste faible et principalement présent dans les zones périphériques et les plus démunies du pays. Selon le ministère de l'Éducation, avant la crise syrienne, le secteur public abritait $30 \%$ des enfants libanais en âge de scolarisation ${ }^{12}$. Aujourd'hui, sur un total de 900000 élèves libanais, seul environ 249000 (pour l'année scolaire 20152016) sont scolarisés dans le secteur public, et le chiffre est en baisse régulière à cause du manque de moyens et des nouveaux besoins éducatifs ${ }^{13}$. La majorité des familles syriennes dépend, pour la scolarisation des enfants, de ce système scolaire public libanais, déjà fortement dévalué et défavorisé. Toujours pour l'année 2015-2016, le nombre d'élèves syriens fréquentant l'école publique s'éle-

6. Karl Blanchet et al., "Syrian refugees in Lebanon: the search for universal health coverage ", in Conflict and Health, vol. $10, \mathrm{n}^{\circ}$ 12, 2016. 7. The United Nations High Commissioner for Refugees (UNHCR), Vulnerability Assessment of Syrian Refugees in Lebanon, 2016, p. 32. Url : https://data.unhcr.org/syrianrefugees/download.php?id=12482. 8. Sarah E. Parkinson, Orkideh Behrouzan, "Negotiating health and life: Syrian refugees and the politics of access in Lebanon ", in Social Science \& Medicine, $n^{\circ} 146,2015$, pp. 324-331. 9. Amnesty International, Agonizing choices: Syrian refugees in need of health care in Lebanon, Londres, Amnesty International; 2014 10. Human Rights Watch, Growing Up Without an Education. Barriers to Education for Syrian Refugee Children in Lebanon, 2016. Url : https://www.hrw.org/sites/ default/files/report_pdf/lebanono716web_1.pdf. 11. Carole Alsharabati, Carine Lahoud, Analysis of Child Éducation Survey, 2016. Url : www.isp.usj.edu.Ib/pdf/Refugees Report USJ 20-Avril 2016. Voir, dans ce numéro, le document de Manuela Casalone. 12. Ministry of Education and Higher Education, Reaching All Children with Education in Lebanon, 2014. Url : http://www.mehe.gov.lb/uploads/file/2015/ Feb2015/Projects/RACEfinalEnglish2.pdf. 13. Isabelle Grappe et al., "L'intégration des élèves syriens dans les écoles libanaises : trois témoignages ", in Confluences Méditerranée, vol. 1, nº 92, 2015, pp. 157-170. 
vait à 158 000, pour 87000 inscrits dans des établissements privés. ${ }^{14}$

C'est surtout à partir de septembre 2015 que les écoles publiques ont commencé à accueillir les écoliers syriens, suite à une décision du ministère de l'Éducation et grâce au financement international. $20 \%$ des écoles publiques, à savoir 238 établissements, ont alors mis en place un horaire de l'aprèsmidi et du soir pour les enfants syriens ${ }^{15}$, l'horaire du matin étant destiné principalement aux enfants libanais. En dépit de ces mesures, à peu près 250000 enfants syriens ne sont toujours pas scolarisés, et parmi eux, le nombre de ceux compris dans la tranche d'âge 15 et 18 ans est particulièrement élevé : compte environ 82000 adolescents non scolarisés ${ }^{16}$ et seuls $4 \%$ des enfants âgés entre 15 et 18 ans ont accès à l'enseignement secondaire ${ }^{17}$.

D'autre part, malgré les efforts accomplis pour renforcer le système scolaire public libanais et en dépit du fait que l'État libanais n'exige pas de permis de séjour pour l'enregistrement des enfants syriens, et donc que la scolarisation est formellement dissociée de la situation juridique de la famille, l'accès à l'éducation et le taux d'abandon restent problématiques pour un grand nombre d'enfants syriens.

Les raisons sont, encore une fois, multiples. Une partie des parents a du mal à trouver du travail ou ne parvient pas à assurer un revenu suffisant pour couvrir les coûts liés à la scolarisation (transport, matériel scolaire, etc.). Plus encore, la situation économique extrêmement précaire des familles a pour conséquence que certaines d'entre elles comptent sur le travail de ses enfants pour survivre, ce qui conduit à leur déscolarisation. À cet égard, la différence est particulièrement frappante entre la situation des enfants dans Beyrouth et ses régions environnantes, et celle de ceux qui se trouvent dans la région agricole de la Bekaa où le taux d'enfants qui travaillent (quelle que soit leur nationalité) est beaucoup plus élevé ${ }^{18}$. La distance de l'école a aussi une influence importante sur l'accès aux études et l'abandon scolaire. Selon les résultats d'une recherche menée par une université libanaise, l'abandon scolaire chez les enfants qui doivent se déplacer pour plus d'une heure atteint les $35 \%$, contre $15 \%$ pour ceux qui doivent se déplacer pendant moins de 10 minutes $^{19}$. Les difficultés de l'apprentissage dues à la diversité des programmes et à l'usage de langues étrangères peuvent être également à l'origine d'un décrochage scolaire. En effet, le programme des écoles libanaises se fait dans une grande partie des établissements en français ou en anglais, ce qui est souvent problématique pour des élèves syriens habitués à recevoir l'enseignement exclusivement en langue arabe. Enfin, signalons les questions de racisme et de harcèlement dont les élèves syriens sont parfois victimes de la part de leurs camarades libanais, et qui peuvent conduire à l'abandon Six ans après le du début de la crise syrienne, à peu près 250000 enfants réfugiés en âge de scolarisation (3-18 ans) - sur les 495910 enfants syriens réfugiés enregistrés au HCR - ont accès au système scolaire. scolaire.

Mais cette situation, encore une fois, doit être analysée à la lumière des conditions sécuritaires difficiles et de l'illégalité dans laquelle se trouve une majorité des réfugiés syriens. L'impossibilité de renouveler leur visa, la peur d'être arrêté ou, tout simplement, de se déplacer entravent durablement la capacité des familles à envoyer leurs enfants à l'école.

\section{Les naissances et l'enregistrement des nouveau-nés}

Depuis 2011, un grand nombre d'enfants syriens (100 000 au 30 août 2016) sont nés au Liban ${ }^{20}$. Ces

14. Inter-Agency Coordination, Lebanon. Éducation Dashboard, jan-may 2016. Url : https://data.unhcr.org/ syrianrefugees/download. php? id=11514, consulté 28 février 2017. 15. Human Rights Watch, Growing Up Without an Education. Barriers to Education for Syrian Refugee Children in Lebanon, 2016, op. cit. 16. Ibid. 17. Inter-Agency Coordination, Lebanon. Education Dashboard, op. cit. 18. Carole Alsharabati, Carine Lahoud, op. cit. Le travail des enfants dans l'agriculture en nette hausse au Liban. Voir aussi, par exemple, " I'OIT tire la sonnette d'alarme ", in L'Orient-Le jour, 14 juillet 2016. 19. Carole Alsharabati, Carine Lahoud, op. cit., pp. 18, 24. 20. Ce taux de natalité est presque le double de celui des libanais (40 0000 naissances/an sur 1 million et demi pour les réfugié syriens, 70 ooo / an sur 4 millions pour les libanais). 
naissances soulèvent la question de l'identité et du sort de ces nouveau-nés dont un grand nombre n'a pas de papiers d'identité et, de ce fait, est dépourvu de toute existence légale. Le HCR estimant que $70 \%$ d'entre eux n'ont toujours pas d'acte de naissance ${ }^{21}$.

Les réfugiés syriens affrontent, en effet, maints obstacles pour obtenir des documents d'identification au Liban, et notamment pour établir un acte de naissance dûment enregistré aux registres des statuts personnels libanais quand il s'agit d'enfants nés sur le territoire libanais.

Cette situation, selon le HCR

Les réfugiés syriens affrontent, en effet, maints obstacles pour obtenir des documents d'identification

au Liban, et notamment

pour établir un acte de naissance dûment enregistré

aux registres des statuts personnels libanais quand il s'agit d'enfants nés sur le territoire libanais. et d'autres organisations nongouvernementales actives dans ce domaine ${ }^{22}$, risque de multiplier le nombre de personnes dépourvues d'une identité juridique et potentiellement apatrides ${ }^{23}$.

La procédure administrative requise afin d'établir un acte de naissance au Liban nécessite, tout d'abord, l'obtention d'un certificat de naissance (shahadat wilada) dressé par l'hôpital du lieu de naissance ou par le médecin ou la sage-femme ayant assisté la naissance. Sur la base de ce certificat de naissance, le mokhtar (officier au niveau local) du lieu de naissance, établit un acte de naissance. À ce stade, les parents sont tenus de présenter leurs papiers d'identité et de payer des frais variant entre 30000 et 100000 livres libanaises. L'acte de naissance établi par le mokhtar doit ensuite être enregistré auprès du bureau de statuts personnels relevant du gouvernorat compétent en fonction du lieu de naissance (noufous). L'enregistrement est conditionné à la possession par les parents d'un permis de résidence valide et est suivi d'une inscription au registre des étrangers. L'acte de naissance enregistré auprès de l'administration libanaise doit ensuite être certifié par le ministère des Affaires étrangères en vue de le produire à l'ambassade syrienne et ce, pour établir un acte de naissance syrien qui enregistre l'enfant auprès de l'administration syrienne de l'état civil.

Selon le Norwegian Refugee Council (NRC) et le $\mathrm{HCR}$, cette procédure conditionnée par la possession d'un titre de résidence valide n'est pas réaliste étant donné que la majorité des déplacés en sont dépourvus. Par ailleurs, lorsqu'une naissance n'est pas déclarée dans un délai d'un an, l'enregistrement ne peut être obtenu que par une décision de justice nécessitant la saisine du juge statuant en matière de statuts personnels, ce qui engendre des frais additionnels ${ }^{24}$. Le NRC et le HCR recommandent, par conséquent, de faire établir l'acte de naissance par le mokhtar et de le présenter au bureau de statuts personnels relevant du gouvernorat compétent en fonction du lieu de naissance. L'objectif est de ne pas être tenu à défaut de suivre une procédure judiciaire et de conserver une possibilité d'obtenir un acte dûment enregistré, sans être tenu d'engager une procédure judiciaire.

Il est indispensable de noter, toutefois, que létablissement de l'acte de naissance par le mokhtar n'est pas évident. $97 \%$ de la population déplacée interviewée par le NRC ont pu obtenir un certificat de naissance. Pour $3 \%$ des interviewés, l'obtention de celui-ci a été impossible, l'accouchement ayant eu lieu à la maison et sans la présence du personnel autorisé à dresser le certificat. Et si $78 \%$ ont pu compléter les procédures chez le mokhtar, seuls $19 \%$ ont pu parfaire l'enregistrement auprès du bureau de statuts personnels relevant du gouvernorat compétent en fonction du lieu de naissance, et

21. Gouvernement du Liban, ONU, Lebanese Crisis Response Plan, jnavier 2017, pp. 117-123. Url : Www.alnap.org/pool/files/lebanon-crisisresponse-plan.pdf. 22. Voir Frontier Ruwad Association, Birth Registration Procedures in Lebanon. Practical Manual. Url : https:// frontiersruwad.files.wordpress.com/2015/03/birth-registration-manual english.pdf ; Norwegian Refugee Council, Birth Registration Update: The Challenges of Birth Registration in Lebanon for Refugees from Syria, janvier 2015. Url : https://www.nrc.no/globalassets/pdf/ reports/the-challenges-of-birth-registration-in-lebanon-for-refugees-from-syria.pdf 23. Ibid. 24. Dans les estimations faites par Frontier Ruwad les frais pour les procédures associées au tribunal s'élèveraient à plus de 1000 dollars. Parmi toutes ces dépenses, les examens médicaux sont les plus élevés, surtout parce que le juge, très souvent, demande un test ADN. Ce test, d'ailleurs, n'est pas requis par la législation en vigueur, mais il fait désormais partie de la procédure judiciaire telle qu'entérinée par les tribunaux (Youmna Makhlouf, communication personnelle). Voir Frontier Ruwad Association, op. cit., p. 15. 
seuls $8 \%$ ont réussi à inscrire l'enfant au registre des étrangers.

Si la différence de procédure d'enregistrement au Liban et en Syrie joue un rôle dans les difficultés rencontrées par les réfugiés qui ne possèdent pas toujours les informations relatives à la procédure libanaise, elle est loin d'être le facteur unique qui influence le processus. Les conditions économiques précaires restent l'un des facteurs majeurs qui influencent son aboutissement, tout comme la présence de barrages et de contrôles de l'armée libanaise qui découragent fortement la mobilité des personnes sans documents d'identité ou de résidence valides. Si $86 \%$ des personnes interviewées ont déclaré s'être rendues, pour l'accouchement, dans une structure conventionnée avec le HCR (où, on le rappelle, les frais médicaux sont couverts à $75 \%$, le reste étant à la charge de la famille), de nombreuses naissances ont lieu dans les camps, d'où l'impossibilité d'obtenir un certificat de naissance. De plus, il arrive que les mères syriennes se fassent prêter des documents d'identité par des voisines libanaises afin de bénéficier de la couverture du ministère de la Santé ${ }^{25}$ lors de l'accouchement. Cela entraine des risques juridiques, l'enfant étant enregistré comme étant né d'une autre femme. La situation de vulnérabilité économique et les obstacles à la mobilité se reflètent aussi dans la difficulté, pour beaucoup, de faire établir l'acte de naissance par le mokhtar compétent en fonction du lieu de naissance. Cela expliquerait, en partie, la différence de proportion entre les procédures complétées auprès du mokhtar et celles complétées auprès du bureau de statuts personnels relevant du gouvernorat compétent en fonction du lieu de naissance.

Un autre obstacle qui se présente aux déplacés est le manque de documents d'identité ou l'absence d'un certificat de mariage valide (mariage non enregistré, entrée illégale sur le territoire, non renouvellement du titre de séjour). Cela entrave l'obtention du certificat délivré par le mokhtar ou l'enregistrement au noufous. Il arrive également que le mokhtar ou les employés du noufous exigent un document de résidence valide même si la loi ne le requiert pas pour compléter les procédures. Ici, comme dans d'autres domaines, les pratiques peuvent diverger par rapport aux prescriptions légales.

Afin de contourner les coûts de l'accouchement ou les problèmes posés par la bureaucratie libanaise, certaines personnes décident de rentrer en Syrie pour accoucher ou pour enregistrer le nouveau-né. Il arrive aussi, afin de récupérer la documentation manquante ou de produire de faux documents, qu'elles fassent appel à un simsar (courtier, médiateur) ou qu'elles tentent de se procurer les documents syriens par le biais de connaissances. 